\title{
El Centro Sainsbury de Salud Mental. Los Servicios Forenses de Salud Mental en Inglaterra y el País de Gales
}

\author{
M Rutherford ${ }^{1}$, S Duggan ${ }^{2}$
}

${ }^{1}$ Coordinador de Política del Centro Sainsbury de Salud Mental.

${ }^{2}$ Director de Justicia Criminal del Centro Sainsbury de Salud Mental.

\section{RESUMEN}

El Centro Sainsbury de Salud Mental (Sainsbury Centre for Mental Health) es una organización benéfica fundada en 1985 por la Fundación Caritativa Gatsby (Gatsby Charitable Foundation). El SCMH trabaja para mejorar la calidad de vida de personas con problemas de salud mental influyendo sobre las políticas y prácticas en salud mental y servicios relacionados. El trabajo para mejorar la calidad de atención de salud mental en los centros penitenciarios es un eje central en la labor de SCMH. Este artículo describe algunos aspectos epidemiológicos con respeto a la salud mental de reclusos en Inglaterra y el País de Gales y los servicios y prestaciones forenses disponibles para el manejo de este tipo de paciente en el entorno penitenciario.

Palabras clave: Salud Mental, Tratamiento, Instalaciones para Atención de Salud, Recursos Humanos y Servicios, Prisión.

\section{THE SAINSBURY CENTRE FOR MENTAL HEALTH. FORENSIC MENTAL HEALTH SERVICES IN ENGLAND AND WALES}

\begin{abstract}
The Sainsbury Centre for Mental Health (SCMH) is a charity founded in 1985 by Gatsby Charitable Foundation. The SCMH works to improve the quality of life for people with mental health problems by influencing policy and practice in mental health and related services. Working to improve the quality of mental health care for people in prison is one of SCMH main work theme. This paper describes some epidemiological aspects of mental health situation of prisoners in England and Wales and the available forensic facilities to manage this kind of patients in prison.
\end{abstract}

Key words: Mental Health, Therapy, Health Care Facilities Manpower and Services, Prison.

Texto recibido: diciembre 2007

Texto aceptado: febrero 2008

El presente artículo está basado en un Informe Interno del Centro Sainsbury de Salud mental, reproducido con su permiso.

\section{INTRODUCCIÓN}

El número de personas en los centros penitenciarios que padecen de problemas de salud mental es muy elevado. Hay estudios que han demostrado que la frecuencia de enfermedades mentales entre la población penitenciaria es tan elevada que afecta a nueve de cada diez reclusos ${ }^{3}$. El Gobierno ha reconocido que "se- guimos encarcelando demasiadas personas con problemas de salud mental" 4 . A la vez, la población penitenciaria ha llegado a niveles sin precedentes. Llegó a un nivel record de 81.106 a finales de mayo del $2007^{5}$.

El Centro Sainsbury de Salud Mental cree que la derivación a los recursos extrapenitenciarios es eficaz para muchos reclusos, y que se beneficiarían de tratamiento fuera del sistema de justicia criminal. La deri- 
vación puede asegurar que personas con problemas de salud mental que cometen ofensas reciban tratamiento en entornos que se adecuen a sus necesidades. Puede también ayudar a reducir la creciente población penitenciaria y en la reducción de las tasas de reincidencia para los que están en libertad, con lo cual ayudará a proteger a la comunidad y ahorrará dinero que de otro modo se invertiría en atender a los reincidentes.

Los servicios forenses de salud mental juegan un papel muy importante en el proceso de derivación a los recursos extrapenitenciarios, sobre todo derivando personas que están a punto de entrar o que ya están en la cárcel. Los servicios forenses proporcionan una detención segura en hospitales especiales donde se hace más énfasis en la atención y el tratamiento que en el castigo. Sin embargo, la información sobre servicios forenses sólo es una parte, y para un conocimiento global, se requiere información de muchas fuentes distintas. Como consecuencia, el conocimiento político y público del tema es escaso, y por lo tanto el debate sobre este asunto es escaso y mal fundamentado. Este documento recoge los hechos y cifras más relevantes para incrementar el conocimiento del tema e informar mejor el debate.

La población penitenciaria ha crecido en la última década, y con ella la de los servicios forenses. $\mathrm{Ha}$ crecido el número de personas detenidas en los servicios forenses a lo largo de esta década, aumentando de 2.650 en 1997 a casi 4.000 en julio de 2007. Además, el número de personas derivadas del centro penitenciario al servicio forense se incrementa cada año, a pesar de los largos retrasos en la derivación desde los centros penitenciarios (CP) a un hospital forense. La población ingresada en los servicios forenses aumentó en un $45 \%$ en diez años entre 1996 y 2006. La mayoría de las personas ingresadas en servicios forenses son hombres adultos jóvenes, pero la edad promedio es más alta que en la población reclusa. Uno de cada ocho internos de la población de los servicios forenses son mujeres, dos veces más que la población femenina en los CP. La mayoría de las personas en servicios forenses cumplen sentencias de cinco años o menos, pero también hay más del $25 \%$ con condenas de más de diez años.

Los tipos de delito son diversos, pero casi la mitad de la población está bajo custodia por delitos sexuales o de violencia. A pesar de la naturaleza grave de muchos de los delitos cometidos por personas en servicios forenses, y un aumento en el número de personas que reinciden durante la libertad condicional, el porcentaje de personas que vuelven a cometer delitos es muy bajo en comparación con el resto de reclusos en libertad.
Este documento se centra en los hospitales forenses de alta y media seguridad, donde se reciben la gran mayoría (casi 97\%) de las personas derivadas por "orden de contención" directamente del CP a los servicios forenses. Debe tenerse en cuenta que las personas bajo acusación de un delito también pueden ser detenidas por la Ley de Salud Mental (Mental Health $A c t$ ) durante el juicio, y pueden ser derivadas a otros servicios de salud mental tales como las unidades de cuidados intensivos psiquiátricos (psychiatric intensive care units o PICUs) o los servicios forenses de salud mental en la comunidad. No se incluyen en este documento a servicios penitenciarios de salud mental como los servicios In-reach o Unidades de Trastorno de Personalidad Grave y Peligrosa (DSPD units). El enfoque de este trabajo es sobre los servicios forenses para adultos, y por lo tanto no incluye información sobre Servicios de Salud Mental para niños y adolescentes (Children and Adolescent Mental Health Services o CAHMS).

Este documento ofrece una visión en forma de boceto de la población actualmente bajo detención. Se plantea una serie de preguntas:

¿Son los servicios forenses una forma apropiada de derivación?

¿Serían más apropiadas intervenciones sociales basadas en la comunidad?

$¿$ Se deben extender de forma masiva los servicios forenses para mejor servir a los miles de reclusos en los CP con trastornos mentales que no reciben tratamiento, o debe mejorarse la atención a la salud mental en prisión?

¿Debe imponerse un límite de tiempo para que las personas sean detenidas en los servicios forenses?

$¿$ Debe vincularse la detención con la seriedad del delito cometido o hasta que el tratamiento haya terminado?

Esperamos que estas cuestiones reciban la atención que requieren de los responsables de formular las relevantes políticas.

\section{1. ¿QUÉ QUIERE DECIR “SERVICIOS FORENSES”?}

En este documento, servicios forenses refiere a los servicios que proporcionan atención a delincuentes con trastornos mentales: personas que han estado en contacto con el sistema de justicia criminal y que se han trasladado a hospitales psiquiátricos forenses.

Hay dos niveles principales de seguridad en los hospitales forenses a los que, tanto delincuentes bajo acusación de un delito, como reclusos pueden de- 
rivarse: alta y media seguridad. También existe un nivel bajo de seguridad, pero muy pocos delincuentes son derivados a estos centros desde los CP o los juzgados. La gran mayoría de estos casos son derivados en principio a las instalaciones de alta o media seguridad. Por lo tanto en este documento "servicios forenses" se refiere a los hospitales de alta y media seguridad. Hoy en día hay casi 4.500 "plazas forenses" (camas) en los servicios forenses de alta y media seguridad.

\section{Alta seguridad}

Todas las camas de alta seguridad se proporcionan por el Servicio Nacional de Salud (NHS). Hay tres proveedores del NHS (los Hospitales de Ashworth, Broadmoor y Rampton), con alrededor de 800 camas de alta seguridad en total. En julio de 2007, 653 de estas camas estaban ocupadas. Las camas de alta seguridad son diseñadas para pacientes detenidos bajo la Ley de Salud Mental 1983, que "representan un grave e inminente peligro para el público". No obstante, un informe en 2000 apuntó que algunos pacientes en los hospitales de alta seguridad no necesitaban ese nivel de seguridad, y debían derivarse a un entorno menos controlado. Como consecuencia 400 pacientes fueron a plazas de niveles inferiores de seguridad antes de $2004^{6}$.

\section{Media Seguridad (ver Anexo)}

Las camas de media seguridad son proporcionadas por el NHS y el sector privado (el segundo proporciona cerca del $35 \%$ de las camas en la categoría de media seguridad). Hay alrededor de 3.500 camas en total en zonas distintas del país. Un informe en 2006 sobre servicios especializados en atención de salud mental señaló que "la distribución de servicios del NHS y del sector privado sigue siendo muy desigual en todo el país"'. Estas plazas están pensadas para pacientes detenidos bajo la Ley de Salud Mental 1983, que "representan un peligro serio para el público". La seguridad y calidad de los servicios de media seguridad se están evaluando por parte del Departamento de Salud, y los resultados se publicaran dentro de poco.

\section{Baja Seguridad}

Las camas de baja seguridad son proporcionadas por el NHS y el sector privado para pacientes detenidos bajo la Ley de Salud Mental 1983, que representan "un peligro significativo para ellos mismos o para otros". Normalmente, pasan a baja seguridad después de un periodo en media seguridad. También pueden utilizarse para pacientes voluntarios. Sin embargo, no existen normativas definitivas en cuanto a lo que constituye o no un tratamiento de baja seguridad, y algunos servicios en este sentido no se distinguen de otros en residencias para "personas con enfermedades mentales en recuperación”.

El máximo tiempo de estancia recomendado en un entorno de baja seguridad son ocho semanas. Las camas de baja seguridad se utilizan en su mayoría para personas detenidas directamente en la comunidad o que han sido trasladados desde instalaciones de media seguridad. De los pacientes derivados entre abril de 2005 a marzo de 2006 , solo el $2 \%$ de los reclusos con sentencias, y el $1 \%$ de los reclusos que esperaban sentencia fueron derivados directamente del CP a instalaciones "abiertas" del servicio forense ${ }^{8}$.

\section{Servicios de Media Seguridad Creados para Mujeres (Women's Enhanced Médium Secure Services)}

Los servicios forenses del NHS cuentan con los nuevos WEMSS disponibles en Londres, Leicestershire, y en el noroeste 9 . La más grande de estas instalaciones es The Orchard, una unidad de 60 camas en Ealing, que proporciona 45 camas para WEMSS y 15 camas de media seguridad. (...)

Los WEMSS están diseñados concretamente para mujeres que necesitan niveles aumentados de intervención y tratamiento dentro de un entorno de media seguridad y para las cuales los servicios actuales de media seguridad no son apropiados. Las pacientes son ingresadas en unidades de alta y baja seguridad, $\mathrm{y}$ en unidades de cuidados intensivos psiquiátricos. El servicio del Orchard tiene un modelo claro de atención como base del tratamiento de mujeres en un entorno (...) lo menos restrictivo posible, y con un enfoque en la planificación dinámica de la atención y el compromiso activo $^{10}$.

\section{LA POBLACIÓN EN SERVICIOS FORENSES}

\section{Número}

El número total de personas detenidas en los servicios forenses se ha ido incrementando a lo largo de los años durante más de una década. Había 3.658 personas detenidas en los servicios forenses al final del año 2006, un aumento del $7 \%$ en comparación con 2005. En julio de 2007 el número había llegado a 3.723 , el mayor hasta la fecha. 


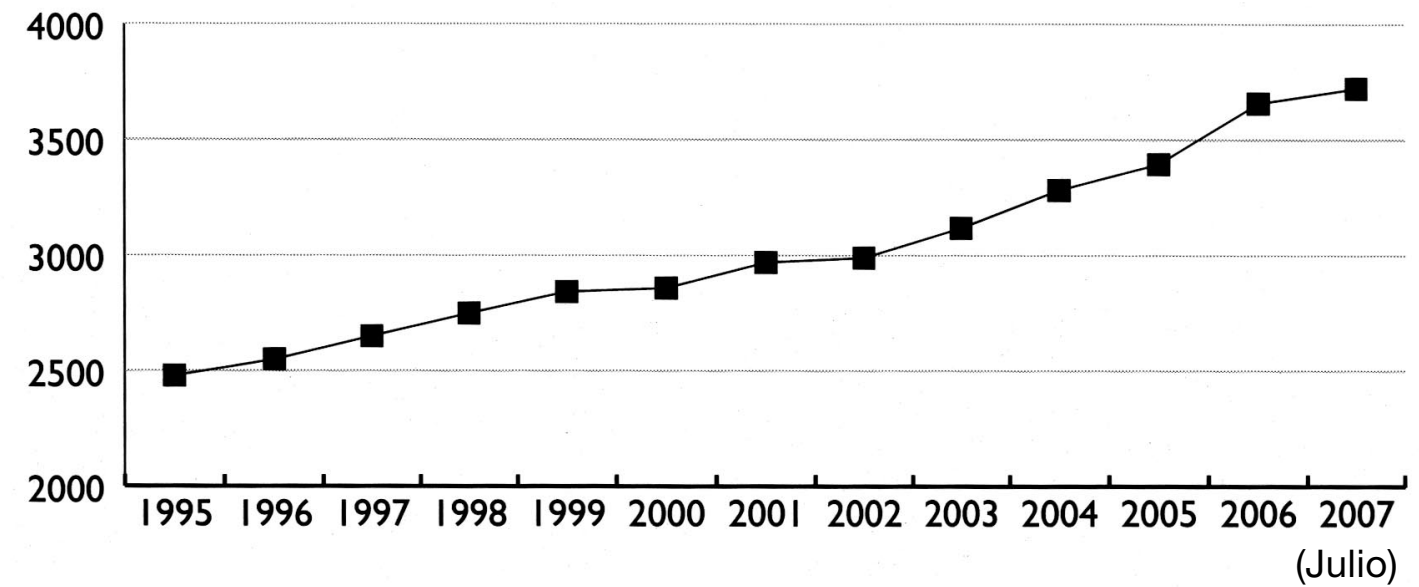

Fig I: La población ingresada en los servicios forenses, 1995-julio de 2007

\section{Genero}

En julio de 2007, la población ingresada en los servicios forenses fue del $88 \%$ en hombres y del $12 \%$ en mujeres. La población femenina es proporcionalmente más alta en los servicios forenses que en los centros penitenciarios. En julio 2007, el 12\% de la población en servicios forenses fueron mujeres (458 en total) en comparación con $6 \%$ en el entorno penitenciario (4.508).

La proporción de mujeres en la población en servicios forenses se ha mantenido estable entre 1995 y 2007. Sin embargo, en los últimos años la población femenina en el entorno penitenciario se ha incrementado proporcionalmente más rápido que la población masculina.

\section{Edad}

En julio de 2007, el $84 \%$ de la población de los servicios forenses tenía entre 26 y 64 años de edad, el $11 \%$ tenían 25 años o menos, y el 4\% tenían más de 65, incluyendo 14 personas con más de 80 años.

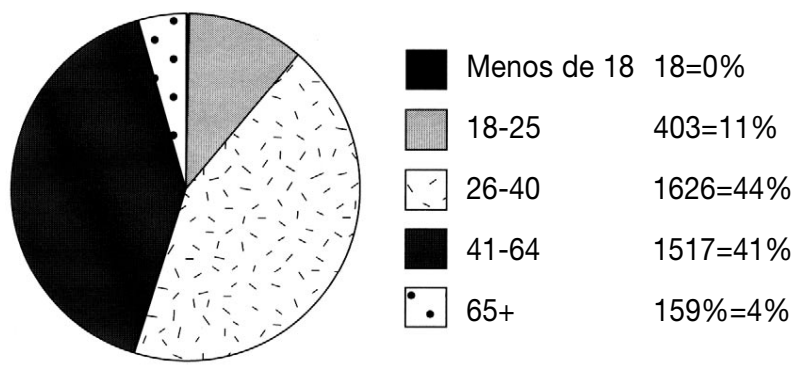

Fig. 2: Pacientes bajo “orden de contención” en el hospital según edad, julio de 2007.
Hay muy pocas personas con menos de 18 años de edad en los servicios forenses. Solo había 18 personas de esta edad en la población en julio de 2007. Sí compararnos, en mayo de 2007, había 2.450 niños de 15-17 años de edad en instituciones para delincuentes menores de edad.

\section{Orígenes étnicos}

La información sobre orígenes étnicos se extrapoló del "Cuéntame en el Censo 2006" (Count Me In Census 2006) de la Comisión de la Ley de Salud Mental, publicado el 23 de marzo de 2007.

El 29\% de las personas de los servicios forenses que no habían sido derivados directamente de la cárcel después de una sentencia de custodia ${ }^{11}$ no eran de origen étnico "británico blanco". $12 \%$ eran de origen "negro o británico negro", y 4\% eran de origen "asiático" o "asiático británico", el 56\% de los pacientes derivados mientras esperaban juicio o estaban en prisión provisional (Sección 48, Ley de Salud Mental 1983) no eran de origen "blanco británico". El 20\% quedaron anotados como "negro o negro británico" y el 7\% como "asiático o asiático británico".

Una tercera parte de los pacientes que habían sido derivados directamente desde del juicio (Sección 37, Ley de Salud Mental 1983) no eran de origen étnico "blanco británico". El 18\% eran de origen "negro" o "negro británico" y el 4\% de origen "asiático" o "asiático británico".

En contraste, las últimas cifras que hacen referencia a las poblaciones penitenciarias muestran que $25 \%$ de los reclusos son de origen étnico "no blanco", el 6\% eran "asiático" o "asiático británico" y $15 \%$ "negro” o "negro británico"12. Según el último censo de 
la población del Reino Unido, 7,9 de los residentes en el país son de un grupo no-blanco, el $4 \%$ son de origen "asiático" o "asiático británico y el $2 \%$ de origen "negro” o "negro británico" Censo ONS 2001. Estas estadísticas resaltan la sobre-representación de las minorías étnicas tanto en los servicios penitenciarios de salud mental como en su equivalente en los servicios forenses.

\section{Diagnóstico}

Todos los pacientes que han sido derivados a los servicios forenses tienen un "trastorno o discapacidad mental”. La mayoría, un poco más de tres cuartos, se diagnosticaron con una enfermedad mental, con o sin otros trastornos.

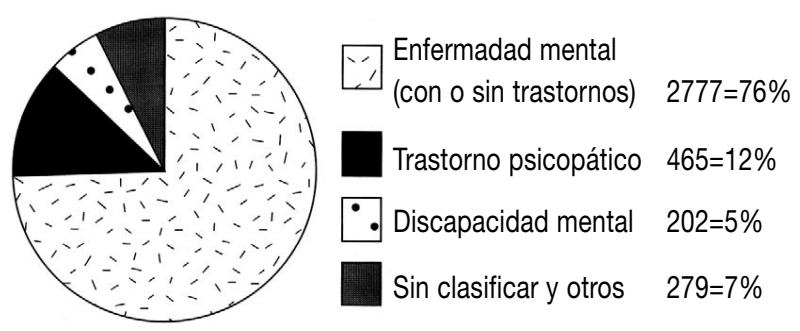

Fig. 3. Pacientes bajo “orden de contención” según tipo de trastorno mental, julio 2007.

Una mayor proporción de mujeres con trastorno mental parece diagnosticarse parece diagnosticarse con trastorno psico. En 2005, el 12\% de hombres detenidos tenían este diagnóstico en comparación con el $21 \%$ de mujeres bajo "orden de contención" en el hospital con un trastorno mental.
Sin embargo, parece que en el hospital una proporción más elevada de hombres detenidos tienen un diagnóstico de enfermedad mental en comparación con mujeres detenidas (...) (70\% comparado con $51 \%$ en 2005).

\section{INGRESOS Y DERIVACIONES}

Los ingresos en los servicios forenses pueden ocurrir en una variedad de formas y por una variedad de caminos. Tres de los más comunes son:

- Derivación a servicios de seguridad directamente del centro penitenciario. Una vez derivado, la duración de la sentencia original de custodia solo es válida si el tratamiento se termina antes de la sentencia. En este caso, el individuo vuelve al centro penitenciario. Derivaciones del CP también ocurre para los que están en custodia provisional o esperando sentencia.

- Un juzgado puede dar a un delincuente con tras-torno mental una orden de hospitalización restringida o sin restricción como alternativa al CP. Estas órdenes tienen su origen en la Sección 37 de la Ley de Salud Mental 1983. Las órdenes restringidas pretenden asegurar que la persona continúe recibiendo un seguimiento y un tratamiento después de salir del hospital. Los pacientes sin restricción sólo se manejan por médicos clínicos y los pacientes con restricción están sujetos a ciertas cautelas por parte del Secretario del Estado de Justicia.

- Los pacientes pueden derivarse a los servicios forenses directamente de los servicios de salud mental de la comunidad.

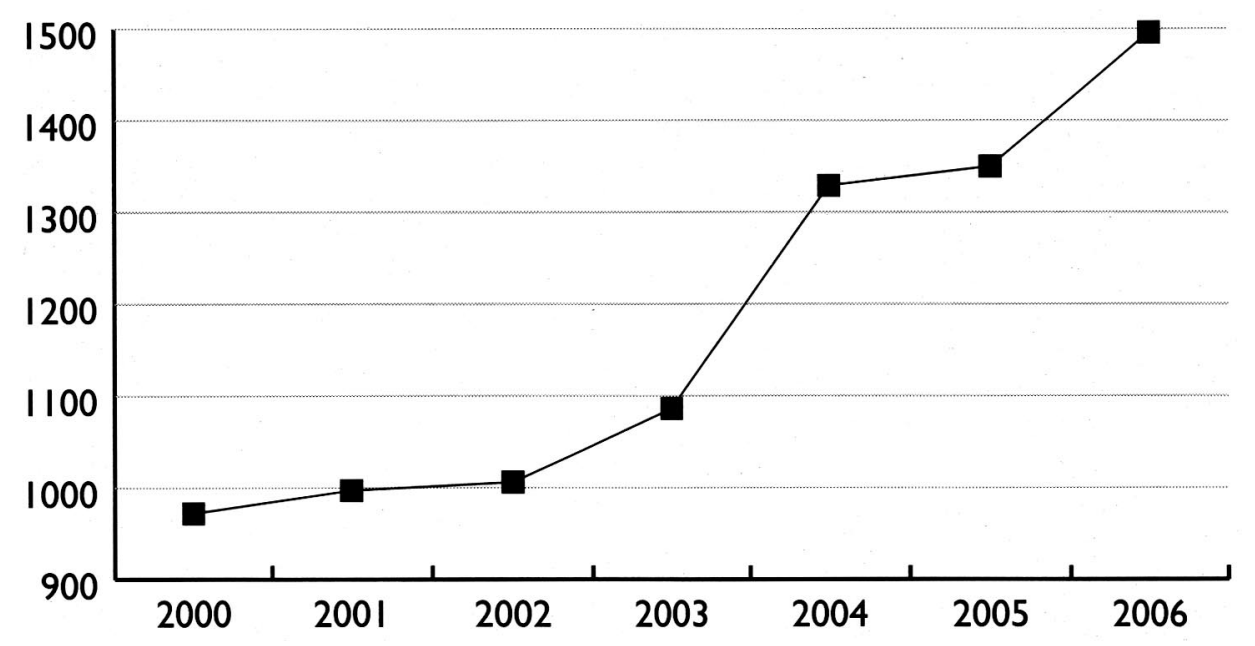

Fig. 4: Ingresos anuales en los servicios forenses, 2000-2006. 


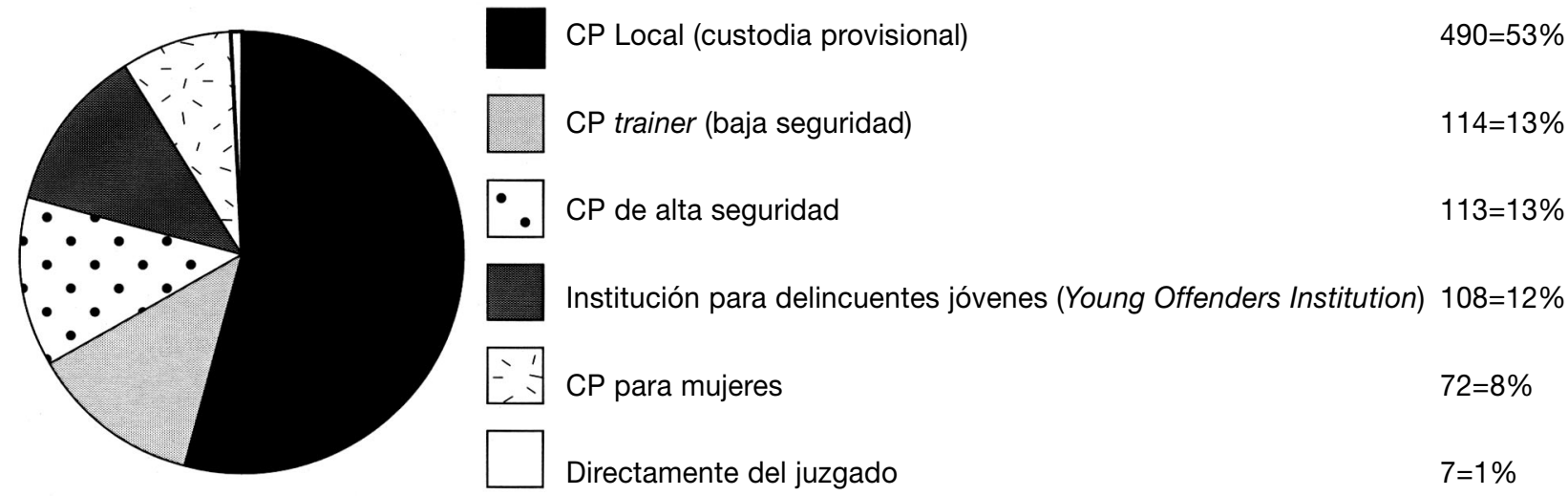

Datos, entre abril 2005-marzo 2006, sin publicar

Fig. 5: entorno penitenciario del paciente derivado desde abril 2005-marzo 2006.

1.496 personas fueron ingresadas en servicios forenses en 2006, un incremento del $11 \%$ de los 1.350 en 2005 y un aumento de $54 \%$ desde 2000 , cuando se ingresaron 972. Los nuevos ingresos representaban el $41 \%$ de la población ingresada en servicios forenses en 2006. El número global de ingresos a los servicios forenses ha ido creciendo a lo largo de los años. El plan del NHS anunció la provisión de 500 camas nuevas de seguridad para servicios forenses. El informe comenta que "modernizar los servicios de salud mental es una de las prioridades claves del Gobierno. Ya hemos puesto en marcha un plan de diez años para establecer nuevos criterios de atención. Las recientes inversiones nos permitirán crear, antes de abril del próximo año, casi 500 camas seguras más, mas de 320 camas atendidas durante las 24 horas del día, 170 equipos de atención en la comunidad (assertive outreach teams) y acceso a servicios las 24 horas del día, los siete días de la semana, para cualquier persona con necesidades complejas de salud mental"13.
El número de personas ingresadas en servicios forenses directamente desde la cárcel (bajo Sección 47 de la Ley de Salud Mental 1983) está aumentando. Se trasladaron a 961 personas a los servicios forenses directamente de la cárcel en 2006, un incremento del $15 \%$ en comparación con las 834 del 2005.

Casi el $50 \%$ de las derivaciones desde la cárcel a los servicios forenses se hicieron mientras los reclusos estaban bajo custodia provisional en cárceles locales (bajo Sección 48 de la Ley de Salud Mental 1983). En 2006, el 49\% (473) de los ingresos en los servicios forenses del CP fueron personas sin sentencia, en custodia provisional, mientras el 51\% (488) fueron reclusos derivados desde el CP después de ser sentenciado.

El número ingresado bajo "órdenes de hospitalización restringida" se ha mantenido estable en los últimos años. En 2006 había 293 ingresos de este tipo (23\% del ingreso total). Las personas detenidas bajo una "orden de contención" (Sección 37 de la Ley de Salud Mental 1983) normalmente han cometido una

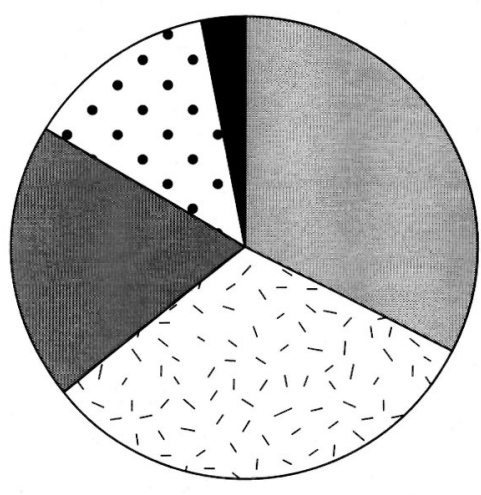

Derivado al hospital después de sentencia

$488=32 \%$

- Derivado del CP sin sentencia/bajo custodia provisional

Orden de hospitalización con orden de contención

- Re-encarcelado después de alta condicional

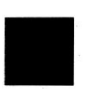

Incapaz de defenderse, no culpable por motivo de enfermedad mental, orden de hospitalización y contención (hospital and limitation direction)
$473=32 \%$

$293=20 \%$

$197=13 \%$

$45=3 \%$

Fig. 6: Pacientes bajo orden de contención ingresados el hospital según categoría jurídica, 2006. 


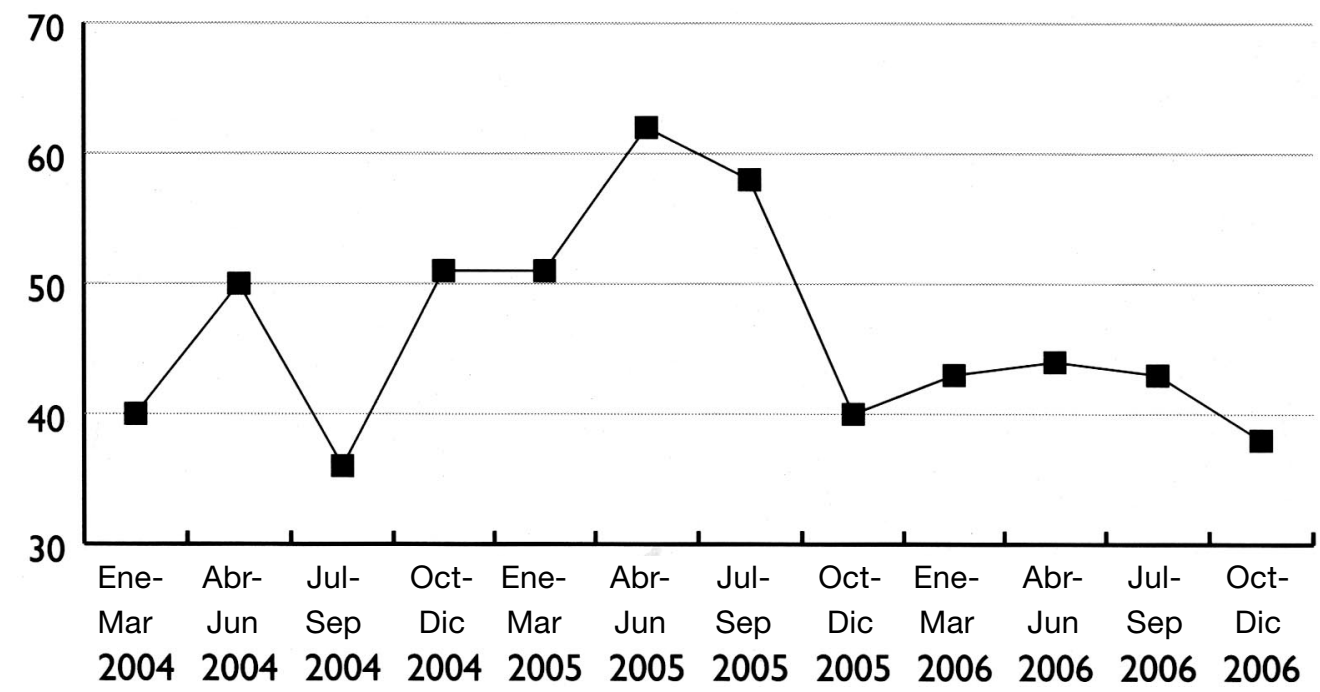

Fuente: Departamento de Salud, Estadísticas de Derivaciones en Salud Mental Penitenciaria, abril 2006, sin publicar.

Fig. 7: numero de reclusos al final del trimestre esperando más de 3 meses para derivación, 2004-2006.

infracción que conlleva una sentencia de custodia pero como resultado de un trastorno mental, no son encarcelados sino que reciben una orden de ingreso en un hospital para tratamiento, sin límite de tiempo.

Menos personas (...) ingresan directamente en los hospitales de alta seguridad. El $8 \%$ de los ingresos en los servicios forenses se hicieron en hospitales de alta seguridad (112) en 2005, una reducción del $10 \%$ en comparación con 2004 (130 de 1.329).

\section{Tiempo de espera para derivación}

Un promedio de 42 reclusos por trimestre esperan más de tres meses para su derivación a los servicios forenses en 2006.

Se descubrió en un estudio de un CP en Londres que después de la toma de decisión de derivar un recluso del CP a los servicios forenses, hubo una espera promedio de 53 días para la derivación. El British Medical Journal comentó que "factores conocidos que contribuyen a este fracaso son por ejemplo la falta de derivación a recursos extrapeniteciarios por parte de los servicios judiciales y la policía, escasa atención en la comunidad, y la presión sobre camas en las unidades de cuidado intensivo psiquiátrico y las unidades de media seguridad. Otros factores menos reconocidos son la falta de recursos para reducir el nivel de contención (stepdown) y la escasez de procesos adecuados de seguimiento de la atención en la comunidad (aftercare), con el factor añadido del bloqueo y retraso en disponibilidad de camas en las unidades de media seguridad ${ }^{14-17}$.
Algunos reclusos en un $\mathrm{CP}$ que reciben la recomendación de derivación a los servicios forenses no son aceptados y se les mantiene en el CP. Cuando una solicitud de derivación no se acepta después de una segunda evaluación por un médico especialista en psiquiatría que procede de un servicio exterior, en el $33 \%$ de las ocasiones es porque el recluso no "cumplía con los criterios para derivación bajo la Ley de Salud Mental 1983". Cuando el recluso no es aceptado para derivación, un $55 \%$ de ellos se manejan en el "ala principal" del CP, y el $35 \%$ en la unidad de ingresos del $\mathrm{CP}^{18}$.

Cuando se decide derivar un paciente, la falta de disponibilidad de camas es la razón más común por retrasos en las derivaciones $(73 \%)$. Sin embargo, en junio de 2007, el Gobierno afirmó que "ha habido una reducción significativa en el número de personas que esperaban más de 12 semanas (...) una derivación - en el trimestre enero-marzo de 2007, cuarenta reclusos (que llevaban más de 12 semanas esperando) estaban en espera, 11 menos que los 51 reclusos que esperaban en el mismo trimestre del $2005^{19}$.

\section{DURACIÓN DE ESTANCIA}

Esta sección utiliza como referencia las "Estadísticas de Delincuentes con Trastorno Mental 2004”. Estos datos son los más recientes sobre los servicios forenses y el tiempo de estancia de los pacientes. 


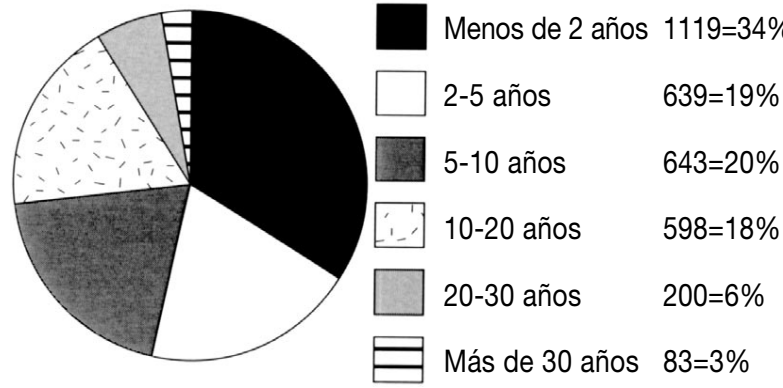

Fig. 8: Duración de la estancia de los pacientes ingresados en los servicios forenses, 31 diciembre 2004.

Nota: en la figura 8, la categoría "menos de 2 años" incluye aquellos que tienen la clasificación de puestos en libertad cuando estaban "sin sentencia o esperando sentencia". Casi la mitad de las 192 personas que abandonaron los servicios forenses mientras tenían esta categoría fueron dados de alta en menos de tres meses 93 (48\%). En cuanto el resto, 47 (25\%) estuvieron entre tres y seis meses, mientras $52(27 \%)$ fueron detenidos durante mas de seis meses.

Más de la mitad (53\%) permanecen en los servicios forenses durante menos de cinco años. El 34\% salen en menos de dos años.

Uno de cada cinco (...) pacientes ingresados se quedó entre cinco y diez años. El 18\% fueron detenidos (...) entre diez y veinte años, mientras el $6 \%$ fueron detenidos (...) entre veinte y treinta años. El $3 \%$ de las personas ingresadas en los servicios forenses con sentencia firme permanecieron durante más de 30 años.

Con respeto a los pacientes con un diagnóstico de "trastorno psicopático", el 50\% habían pasado más de diez años en un hospital, en comparación con el $33 \%$ de los pacientes con algún tipo de "discapacidad mental", y solo el $25 \%$ de los pacientes con una "enfermedad mental” (con o sin "otros trastornos").

Una vez derivado desde un CP a un hospital forense, la sentencia original del paciente es irrelevante en cuanto a su estancia en los servicios forenses. Esa sentencia sólo vuelve a ser relevante si la persona que ha sido derivada vuelve a la cárcel después de ser puesta en libertad desde los servicios forenses. En ese caso el paciente se quedará en el CP hasta cumplir lo que queda de su sentencia.

\section{TIPO DE DELITO COMETIDO}

Este epígrafe usa como referencia las "Estadísticas de Delincuentes con Trastornos Mentales 2004" (Statistics of Mentally Disordered Offenders 2004) editadas en diciembre de 2005. Estos son los datos más recientes sobre los servicios forenses y categorías de delito. Las cifras se refieren a los delitos que se figuran en la sentencia, no a los que se cometieron mientras el paciente estaba en detención.

El número de personas ingresadas en los servicios forenses en 2004 fue mayor que los ingresos en 2003 en todas las categorías de delito (menos robo y posesión de bienes robados). En 2004 también hubo el mayor número en cada categoría de delito en los últimos 5 años, y en muchos casos también fueron los números más altos de toda la década anterior.

El delito más común cometido por personas ingresadas en servicios forenses en el año 2004 fue "violencia contra la persona" ( $36 \%$ del total). Este porcentaje aumentó en un 20\% desde el año 2003 (398 a 478). Sin embargo, la proporción de ingresos de personas con condenas por violencia contra las personas se había mantenido estable desde 1996.

Los siguientes delitos más comunes fueron "daños criminales" ( $14 \%$, la mayoría por "incendio premeditado") y "robo" (10\%).

El número de personas ingresadas que tienen sentencia de homicidio aumentó hasta el $65 \%$ en los últimos 5 años entre 2000 y 2004 (62 a 102). Por delitos "violentos" pero no mortales, el incremento fue del $46 \%$ durante el mismo periodo (221 a 323).

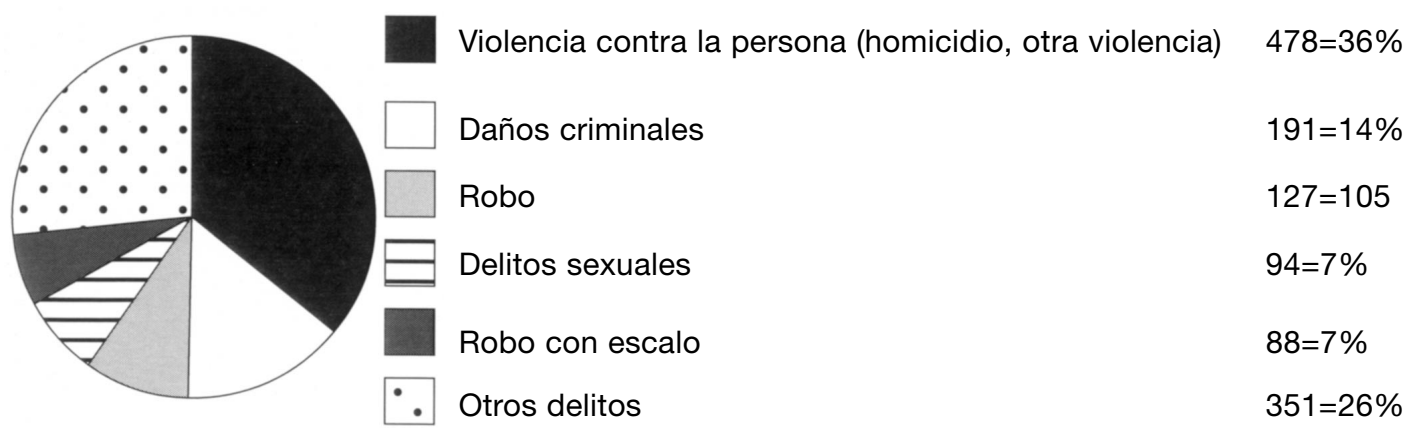

Fig. 9 pacientes contenidos ingresados a los servicios forenses durante 2004, según tipo de delito. 
Entre los pacientes ingresados con 40 años de edad o más, "violencia contra la persona" fue proporcionalmente más común que en otros grupos de edad (33\%). Sin embargo, "robo" fue más común entre los pacientes con menos de 40 años (12\%) que en los pacientes con 40 o más años (3\%). Además, "robo con escalo" fue dos veces más común en el grupo más joven $(8 \%)$ que en el grupo de mayor edad $(4 \%)$.

Ocho personas fueron detenidas en servicios forenses durante 2004 como "inmigrantes detenidos".

\section{ALTAS Y DISPOSICIONES}

Hubo 1.133 altas o derivaciones en pacientes bajo contención de los servicios forenses en 2005, 7\% más que en 2004 cuando hubo 1.060. 469 (o 41\%) de los pacientes dados de alta volvieron directamente a la comunidad extrapenitenciaria.

El alta o derivación más común en 2005 fue "alta condicional en la comunidad" (32\%). El siguiente fue "dado de alta por decisión judicial, sin volver a la comunidad" (27\%), permaneciendo en el hospital sin contención alguna. El 14\% "permanecieron en el hospital sin contención” (sin decisión judicial), y el 14\% fueron "devueltos al centro penitenciario".

El número de personas dadas de alta para volver directamente a la comunidad desde los servicios forenses se ha incrementado al $38 \%$ entre 2000 y 2005 (340 a 469).

El $76 \%$ de los pacientes dados de alta para volver a la comunidad (357) en 2005 tenían altas condicionales, autorizadas por el Ministro de Estado (Secretary of State) o por un Tribunal de Evaluación de Salud Mental (Mental Health Review Tribunal).

El 10\% de las personas que dejaron los servicios forenses en 2005 volvieron al CP para seguir cumpliendo su condena (113 de los 1.133). Esto supone una reducción del 13\% en 2004 (135 de los 1.060). La cifra incluye 47 reclusos sin sentencia o en custodia provisional que volvieron al centro penitenciario después de darse de alta.

El 25\% (164) de los pacientes dados de alta, aunque no a la comunidad, se quedaron en el hospital. En esta situación ya no están sujetos a sus restricciones previas.

\section{TASAS DE REGRESOS Y REINCIDENCIA}

Ha habido un incremento notable en el número de personas regresadas (recalled) a los servicios forenses. 197 pacientes con "orden de contención" fueron regresados al hospital después de un alta condicional en el año 2006, un aumento del $25 \%$ en comparación con 2004.

Los reingresos representan un $13 \%$ del número total de los ingresos a los servicios forenses. Esta proporción se ha duplicado en los últimos años. En 2000, los reingresos suponían solo un 7\% de los ingresos nuevos (hubo 65 en total). Las tasas de reincidencia para los pacientes dados de alta para entrar en la comunidad desde los servicios forenses fueron muy bajas en comparación con las personas puestas en libertad desde los CP. 843 pacientes fueron dados de alta por vez primera entre 1999 y finales de 2003. De los 717 casos localizados en El Sistema Informático Nacional de la Policía, menos de 1 de cada 10 (7\%) volvieron a cometer un delito en los dos años después de darles el alta. Un $2 \%$ de los pacientes que fueron puestos en libertad y que fueron localizados, reincidieron por delitos sexuales o por delitos de violencia. Esta cifra se compara con la tasa global de reincidencia de reclusos adultos en los dos años siguientes a su puesta en libertad, por delitos sexuales $(27 \%)$ y delitos de violencia $(46 \%)^{20}$.

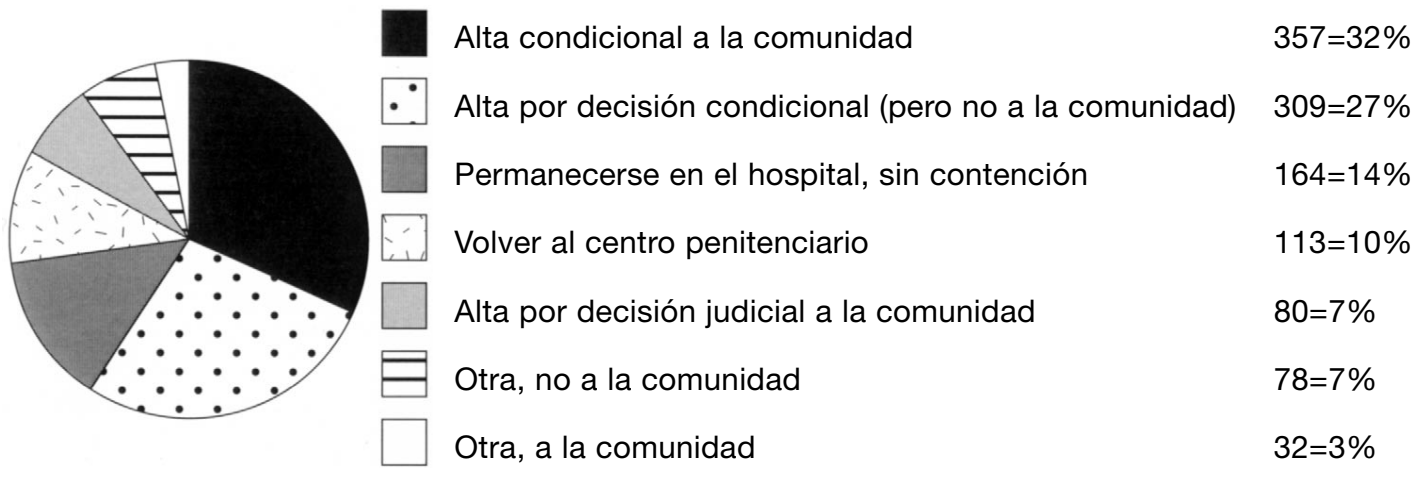

Fig. 10: Altas puestos en libertad de pacientes en servicios forenses, 2005. 
Es importante tener en cuenta lo difícil que es sacar conclusiones cuando se hacen comparaciones entre las cifras de reincidencia de los reclusos puestos en libertad y los pacientes dados de alta de los servicios forenses. Algunas posibles razones que explican el nivel relativamente bajo de reincidencia de los pacientes dados de alta de los servicios forenses pueden ser:

- Un seguimiento más cercano de los pacientes dados de alta en la comunidad que el que se hace a los ex reclusos. (Sección 117 de la Ley de Salud Mental 1983).

- La naturaleza del delito original puede haber estado relacionada con la enfermedad mental del paciente, y por lo tanto hay menos probabilidades de que la persona reincida después de haber recibido tratamiento.

- Los ingresos a los servicios forenses incluyen personas bajo acusación de un delito, pero sin recibir condena, así que es posible que no hayan cometido el delito.

- El impacto de algunos medicamentos sobre el estilo de vida después del alta puede reducir conductas reincidentes.

- Algunas personas "dadas de alta" de los servicios forenses se quedan voluntariamente en el hospital, y por lo tanto están menos expuestos a la posibilidad de reincidir.

\section{MORTALIDAD EN SERVICIOS FORENSES}

El número de personas que se murió mientras estaban en servicios forenses cambió poco entre 2000 y 2005 , a pesar de un incremento a nivel general de la población. En 1998, murieron 32 personas mientras estaban en detención, en comparación con 26 personas de los 1.333 en detención en 2005.

Sin embargo, el número de muertes por año en los Centros Penitenciarios ha sido más variable21. En 2000 hubo 142 muertes en custodia, incluyendo 82 suicidios. En 2004 hubo 208 muertes, de los cuales 95 fueron suicidios. En 2006, hubo 154 muertes, y el número de suicidios se había reducido a 67 . La reducción entre 2004 y 2006 se atribuye en gran medida al trabajo de la Unidad de Mayor Seguridad en Custodia (Safer Custody Unit) y los Planes de Evaluación de Atención en Custodia y Trabajo en Equipo [Assessment Care in Custody and Teamwork (ACCT) $]^{22}$.

\section{FUENTES DE DATOS}

Todas las fuentes se refieren a Inglaterra y el País de Gales. Las cifras de este documento proceden de datos del Departamento de Salud y el Ministerio de Justicia (y la antigua Oficina de Asuntos Interiores o Home Office), y de algunas investigaciones de organizaciones independientes. Los datos son los más recientes que hay disponibles de momento, y mientras la mayoría de las cifras son para los años 2006 y 2007, algunos provienen de años anteriores cuando la información fue recogida. Si no se afirma que provienen de otras fuentes, todas las estadísticas de los años 2006 y 2007 provienen de correspondencia personal con la Unidad de Salud Mental de la Oficina de Asuntos Interiores y el Ministerio de Salud. Todas las estadísticas del 2005 y antes (si no se hace referencia a otra fuente) proceden de las "Estadísticas sobre Reclusos con Trastornos Mentales 2005" publicado en marzo de 2007 (esta es la ultima información publicada por el Gobierno con detalles sobre el número de reclusos ingresados, detenidos o dados de alta de los servicios forenses).

\section{AGRADECIMIENTOS}

Los autores agradecen a Linda Seymour (Directora de Política, Centro Sainsbury para Salud Mental) y John Levy (Consejero Independiente a Healthcare) por sus comentarios y sugerencias. Jo Keil (Asistenta de Investigación, Centro Sainsbury) por su apoyo y el concepto del documento.

Agradecimientos especiales a Nigel Bastón y sus colegas en la Unidad de Salud Mental de la Oficina de Asuntos Interiores, y a Vanessa Fowler (Salud Mental del Recluso (Offender Mental Health) Directora de Política de Offender Mental Health, Ministerio de Salud) por proporcionar muchos de los datos recientes.

También, agradecemos a Janet Leech [Directora de Política de Servicios Seguros, Health and Offender Partnerships (NOMS)], a Steven Woolgar (Director de Política y Normativas, Partnerships in Care, y al Profesor John Gunn (Catedrático de Psiquiatría Forense, Instituto de Psiquiatría, King's College, Londres) por sus comentarios y clarificaciones de gran valor. 
El Centro Sainsbury de Salud Mental. Los Servicios Forenses de Salud Mental en Inglaterra y el País de Gales

\section{ANEXO}

\section{Autoridad Regional de Salud}

Camas

Londres

848

(Antes Londres norte central, Londres noreste, Londres noroeste, Londres sureste, y Londres suroeste)

North West

(Antes Cumbria y Lancashire; Cheshire y

Merseyside; y Greater Manchester)

East of England

(Antes Norfolk, Suffolk y Cambridgeshire;

Essex; y Bedfordshire y Hertfordshire)

Yorkshire y The Humber

(Antes North y East Yorkshire y North

Lincolnshire; WestYorkshire; y South

Yorkshire)

East Midlands

(Antes Trent; y Leicestershire,

Northamptonshire y Rutland)

West Midlands

(Antes Birmingham y Black Country;

Shropshire y Staffordshire; y West Midlands

South)

North East

(Antes Northumberland, Tyne y Wear; y

County Durham y Tees Valley)

South Central

(Antes ThamesValley; y Hampshire y Isle

of Wight)

South West

(Antes Avon, Gloucestershire y Wiltshire;

Dorset y Somerset y South West Peninsula)

South East Coast

(Antes Surrey y Sussex; y Kent y Medway)

\section{Total para Inglaterra}

Tabla 1 Capacidad de camas de media seguridad en el NHS según Autoridad Regional de Salud, 2005

(basado en Laing \& Buisson, p. 19).

\begin{tabular}{lcc}
\hline Proveedor & Hospitales & Camas \\
\hline Partnerships in Care & 13 & 748 \\
Care Principles Ltd & 5 & 301 \\
StAndrew's Group & 3 & 224 \\
Priory Group & 2 & 144 \\
Covenant Healthcare Ltd & 3 & 110 \\
Craegmoor Ltd & 4 & 89 \\
Cygnet Health Care & 1 & 82 \\
Alpha Hospitals Ltd & 1 & 42 \\
Otros proveedores & 4 & 87 \\
\hline Total & $\mathbf{3 6}$ & $\mathbf{1 . 8 2 7}$ \\
\hline
\end{tabular}

Tabla 2. Proveedores Principales de Hospitales de Salud Mental incluyendo Servicios de Media Seguridad en el Reino Unido, 2006 (Laing \& Buisson 2006, p. 20)

Nota: estas cifras son los números totales de camas en servicios forenses en hospitales independientes que proveen media seguridad. Incluyen algunas camas de un nivel inferior de seguridad, no solo de media seguridad.

\section{Sector independiente}

La tabla 2 arriba muestra que una proporción elevada de las camas de media seguridad de los servicios forenses son proporcionadas por el sector independiente (privado/no comisionado por el NHS).

Las cifras del año 2006 muestran que había 36 hospitales privados que proporcionan más de 1.800 camas seguras con un cliente de media seguridad de primaria. Partnerships in Care son actualmente el proveedor más grande, seguidos por Care Principles Ltd, St Andrews Group y el Priory Group. Care Principles también tienen cinco hospitales de media seguridad para personas con dificultades de aprendizaje.

\section{REFERENCIAS BIBLIOGRÁFICAS}

1. www.scmh.org.uk consulta Diciembre de 2007.

2. Singleton, N., Meltzer, H., Gatward, R. Psychiatric morbidity among prisoners in England and Wales, London: ONS. 1998.

3. Home Office, A Five-Year Plan for Protecting the Public and Reducing Re-offending, London: Home Office. 2006. 26.

4. Home Office, Population in Custody, Monthly Tables, May 2007 England and Wales, London: Home Office 2007. 5. 
5. Tilt, R., Perry, B., Martin C., et al. Report of the Review of Security at the High Security Hospitals, London: Department of Health. 2000.

6. Laing and Buisson, Mental Health and Specialist Care Services UK Market Report 2006.

7. Department of Health. Comparison of Section 47 and Section 48 Transfers to Type and Security Level of Receiving Hospital/In-patient Unit Between April 2005-March 2006, Documento Interno 2007.

8. Lord Hunt of King's Heath, Hansard Written Answers, Health: Rampton Hospital, II Jun 2007: Column WA235. Documento Interno 2007.

9. see www.theorchard.wlmht.nhs.uk consulta Septiembre 2007.

10. Mental Health Act Commission (2007), Count Me In Census 2006, 23 March 2007, www.mhac. org.uk/census2006/2006Results.php.

11. Home Office. Offender Management Caseload Statistics 2005, London: Home Office. 2006.

12. Department of Health. The NHS Plan: A plan for investment, A plan for reform, London: DH. 2000

13. Sales, B. and McKenzie, N. Time to act on behalf of mentally disordered offenders, in British Medical Journal, 2007; 334: 1222.

14. Rutherford, H. and Taylor, P J. The transfer of women offenders with mental disorder from pri- son to hospital, in Journal of Forensic Psychiatry and Psychology, 2004; 15: 108-23.

15. Senior, J. and Shaw. J. Prison healthcare, in Jewkes, Y (ed.), Handbook on Prisons, Collompton. Willan Publishing. 2007.

16. Birmingham, L. The mental health of prisoners, in Advances in PsychiatricTreatment.2003; 9: 1919

17. Department of Health, Prison Mental Health Transfers:An Audit Report, October 2006, unpublished.

18. Winterton, R., Hansard Written Answers, Health Services: Prisons, 27 June 2007, Column 821 W.

19. Home Office. Re-offending of adults: results from the 2004 cohort, 06/07, London: Home Office. Documento Interno. 2007.

20. Ver www.inquest.gn.apc.org/data deaths-in-prison.html consulta septiembre 2007.

21. Ver www.hmprisonservice.Qov.uk/adviceandsupport/prison-life/selfharm/ consulta septiembre 2007.

\section{CORRESPONDENCIA:}

Max Rutherford. max.rutherfordC@scmh.org.uk.

Sean Duggan.

sean.duggan_scmh.org.uk. 